\title{
Calculation of Fermi level location and point defect ensemble in CdTe single crystal and thin films
}

\author{
V.V. Kosyak, A.S. Opanasyuk \\ Sumy State University \\ 2, Rimsky-Korsakov str., 40007 Sumy, Ukraine \\ Phone: 38(0542)392237,fax: 38 (0542) 334058, e-mail: opanasyuk@ua.fm,v_kosyak@ukr.net
}

\begin{abstract}
With the use of expressions obtained from the "first principles", the ensemble of point defects was calculated, and the location of a Fermi level in undoped cadmium telluride single crystals and thin films depending on physico-technological conditions of their fabrication and annealing is determined. The model in use accounts the most complete spectrum of defects in chalcogenide, including defects in the cadmium and tellurium sublattices, and the existence of an antistructural defect on the cadmium sublattice. Calculations of the concentration of neutral and charged defects are realized for two extreme cases - full equilibrium and quenching. The comparison of the obtained results with the data of modeling provided with the use of a quasichemical formalism for a number of models most used presently is carried out. It is shown that all models describe well the results of Hall measurements of the concentration of free carriers in single crystals in the range of high cadmium pressure, but give essentially different results in the range of high tellurium pressure. Dominant defects in single crystals at high cadmium pressure and annealing temperatures are twice charged tellurium vacancies or interstitial cadmium atoms, which is in agreement with experimental results, as just such defects can provide the dependence of the concentration of free carriers on cadmium pressure as $n \sim P_{\mathrm{Cd}}^{1 / 3}$. A type of defects which are dominant in a tellurium-enriched material is determined by the chosen model. This allows us to make conclusions about the validity of the considered models and to specify the thermodynamic parameters of the defect creation processes in a material.

The offered model can be used for modeling the ensemble of point defects in any $A_{2} B_{6}$ compounds. Thus, the problem of the choice of models adequate to experimental data is reduced to the determination of the creation energy for uncharged defects and the depth of energy levels of charged defects.
\end{abstract}

Keywords: cadmium telluride, point defects, quasichemical formalism, "ab initio" approach, single crystals, thin films.

Manuscript received 04.07.07; accepted for publication 27.09.07; published online 30.11.07.

\section{Introduction}

Due to unique physical properties, CdTe is the object of careful studies during a long time, as a perspective material to produce the detectors of $\mathrm{x}$-ray and gamma radiation, converters of the solar energy, and devices for microelectronics [1]. At the same time, the expectations of the researchers were not substantially justified in connection with the complexity of obtaining the bulk and film CdTe samples with predetermined characteristics. For this reason, the main task of modern scientific researches of $\mathrm{CdTe}$ is the development of technologies of obtaining the single crystals and thin layers with controllable point defects ensemble (PDE), as just the PDE determines electrophysical parameters of a material. The choice of optimum parameters of the influence on chalcogenide is usually realized by modelling the PDE in cadmium telluride in dependence on technological conditions of its obtaining and annealing with the use of the quasichemical formalism [2].

The procedure of modelling is reduced to the solution of a system of equations, which describe the introduction of defects in a solid from the gas phase, jointly with the condition of electroneutrality of a 
material. In case of the formation of defects in the metal sublattice, such quasichemical equations (QE) are as follows:

$$
\begin{aligned}
& A_{A}^{0} \leftrightarrow A_{g}+V_{A}^{-}, K_{V_{A}}=\frac{N\left(V_{A}^{-}\right) P_{A}}{N\left(A_{A}^{0}\right)}, \\
& A_{g} \leftrightarrow A_{i}^{+}, K_{A_{i}}=\frac{N\left(A_{A}^{+}\right)}{P_{A}}, \\
& n+N\left(V_{A}^{-}\right)=p+N\left(A_{A}^{+}\right),
\end{aligned}
$$

where $A_{A}^{0}$ - atom at the sublattice site; $A_{g}$ - atom in the gas phase; $V_{A}^{-}-$monovalent vacancy in the $A$ sublattice; $A_{i}^{+}-$monovalent interstitial atom; $K_{x}-$ constant of $\mathrm{QE} ; N(X)$ - the corresponding concentration of defects; $P_{A}$ - pressure of the $A$ component during the annealing of samples; $n$ and $p-$ free electron and hole concentrations in a material, respectively.

The type and number of the equations describing the equilibrium of defects in a material [3-8] are determined by a model of the creation of defects which is accepted a priori, because the experimental data allow one to determine only a possible type of dominant defects. The constants of QE in system (1)-(3) are given as: $K_{1}=K_{0} \exp \left(-\frac{\Delta H}{k T}\right)$, where $K_{0}-$ constant that does not depend on temperature; $\Delta H$ - enthalpy of the creation of defects; $k$ - Boltzmann constant; $T-$ temperature, at which the reaction runs [2]. The constants can be also determined by the comparison of the results of modeling with those of experimental measurements of the electrical characteristics of chalcogenide at high temperatures $\left(400-1000^{\circ} \mathrm{C}\right)$. As the measurement of the concentration of neutral point defects in a material by standard electrophysical methods is impossible, QE describing the introduction of defects in a solid from the gas phase are written for charged defects. Further, the approximation like $A_{i}^{+} \approx n$ is used. By determining the inclination angle for a straight line on the plot $\lg n-10^{3} / T$ and a segment that is cut by a line on the axis of concentrations, it is easy to find $K_{0}$ and $\Delta H$ [2]. The reaction constants for the creation of defects can be determined more reliably, if the values of $n$ calculated with the help of the given constants are fit to experimental data by several parameters: the inclination of isotherms and isobars, the values of $n$ on isotherms and isobars, and dependences at $P_{\text {Cdmax }}[6]$.

The first set of the QE constants for PDE in CdTe was offered by Nobel [7]. By considering the balance of defects in chalcogenide, he assumed that the creation of defects in a material occurs according to Frenkel's mechanism over the cadmium sublattice. The alternative point of view is the model which is offered by Chern [8]. By this model, a material can contain defects in both the cadmium and tellurium sublattices. During the subsequent researches, the model of defect creation became more complicated, and simultaneously the meaning of QE constants was specified. The basic models that were more often used for the explanation of electrical properties of CdTe were systematized by us in [9].

\section{Models and results}

The traditional approach to the study of PDE in CdTe has a number of basic disadvantages. First, as a rule, the majority of works deals with the calculation of only the concentration of charged defects, though their concentration depends really on both the concentration of neutral centers and the Fermi level location in a material which can depend, in its turn, on the concentration of doped or uncontrollable impurities. Second, the comparison of the results of modeling with experimental data is a difficult task, because, as a rule, the defect creation reactions are considered with application a gas-phase reaction, while the significant range of physical methods allow one to determine the depth of the energy levels of point defects in the band gap (BG) of a solid material.

Recently, the tendency to evaluate the concentration of point defects in various materials by "the first principles" approach [10] is observed. It provides a more correct approach to the description of PDE in a material: first, the concentration of neutral interstitial defects loaded from the gas phase is calculated, and then the processes of their ionization in a solid state depending on the Fermi level location are considered.

We now obtain the basic expressions which are used in such an approach by an example of the formation of a metal vacancy $V_{A}^{0}$. Under full equilibrium conditions, the concentration of any point defect can be expressed through thermodynamic potentials of the defect creation process. According to the law of mass action [11],

$\Delta \mu_{A V}=\mu_{A}+\xi\left(V_{A}^{0}\right)-\xi\left(A_{A}^{0}\right)=0$,

where $\Delta \mu_{A V}-$ change of the chemical potential; $\mu_{A}=\mu_{A}^{0}+k T \ln P_{A} ; \mu_{A}-$ chemical potential of $A$ atoms in the gas phase; $\mu_{A}^{0}$ - chemical potential of $A$ atoms in the gas phase under standard conditions; $\xi\left(V_{A}^{0}\right)=\xi^{0}\left(V_{A}^{0}\right)+k T \ln N\left(V_{A}^{0}\right)-$ chemical potential of a material with point defects; $\xi^{0}\left(V_{A}^{0}\right)$ - chemical potential of point defects under standard conditions; $\xi\left(A_{A}^{0}\right)=\xi^{0}\left(A_{A}^{0}\right)+k T \ln N\left(A_{A}^{0}\right) ; \quad \xi\left(A_{A}^{0}\right) \quad$ - chemical potential of an atom at the sublattice site (i.e., the chemical potential of the perfect crystal); $\xi^{0}\left(A_{A}^{0}\right)-$ 
chemical potential of an atom at the sublattice site under standard conditions

By taking the logarithm of expression (4), we get

$N\left(V_{A}^{0}\right)=\frac{N\left(A_{A}^{0}\right)}{P_{A}} \exp \left(-\frac{\xi^{0}\left(V_{A}^{0}\right)-\xi^{0}\left(A_{A}^{0}\right)+\mu_{A}^{0}}{k T}\right)$.

In modeling the defect creation process under equilibrium conditions between the solid and the gas phase, it is necessary to know the meaning of thermodynamic functions (free energy, Gibbs energy, and chemical potential) for each component. The free energy of the gas phase can be found with the help of the well-known expression

$$
F=-k T \ln Z \text {. }
$$

For a gas which consists of $N$ identical molecules, the thermodynamic potential $Z$ can be expressed as the molecular partition function

$Z=\frac{1}{N !} z^{N}$,

where $z=\sum_{i} \exp \left[-\frac{u_{i}}{k T}\right]$.

The total energy $u_{i}$ of a particle in the $i$-th state can be written as

$u_{i}=u_{0}+u_{\text {trans }}+u_{\text {rot }}+u_{\mathrm{vibr}}$,

where $u_{0}$ is the self-energy in the $i$-th state, and $u_{\text {trans }}$, $u_{\text {vibr }}$, and $u_{\text {rot }}$ are, respectively, the translation, vibration, and rotational components of the total energy.

Then, in accordance with (7), we have

$z=z_{0} \cdot z_{\text {trans }} \cdot z_{\text {rot }} \cdot z_{\mathrm{vibr}}$

For a monatomic gas, $u_{\text {rot }}=u_{\text {vibr }}=0$, and $z_{\text {rot }}=z_{\text {vibr }}=1$. Then it is possible to write down the relation $z=z_{0} \cdot z_{\text {trans. }}$. If $N$ particles with mass $m$ in the gas phase occupy a volume $V$, then

$z_{\text {trans }}=\frac{1}{V}\left(\frac{2 \pi m k T}{h^{2}}\right)^{\frac{3}{2}}$,

where $h$ - Planck constant, and $m$ - mass of a particle.

Let's find the free energy of a particle, by substituting (7)-(10) in (6) and considering that $n N_{a}=N$ and $V=n R T / P$ :

$F^{\text {trans }}=n u_{0}-n k T(\ln K+1)$,

where $K=(2 \pi m)^{3 / 2} \frac{(k T)^{5 / 2}}{h^{3}}$.

The Gibbs energy is connected with the free energy by the expression $G=F+n k T$. Then we have $G^{\text {trans }}=n u_{0}-n k T(\ln K)$.
By using the definition $\mu=\left(\frac{\partial G}{\partial n}\right)_{P, T}$, it is possible to find the chemical potential of a particle in the gas phase:

$\mu_{A}=u_{0}-k T(\ln K)$.

After simple transformations, we finally obtain

$\mu_{A}=\mu_{A}^{0}+k T \ln P_{A}$,

where $\mu_{A}^{0}=u_{0}-k T(\ln K)$.

By considering the thermodynamic functions of defects in a solid, we have no right to use the relation $z_{\text {rot }}=z_{\text {vibr }}=1$. On the contrary, the basic contribution to $u_{i}$ will be brought by the vibration energy of a particle: $\quad u_{i} \approx u_{\text {vibr }}$, because $u_{\text {rot }}=u_{\text {trans }}=0$. Therefore, it is necessary to research the complex processes of interaction of an atom located in the crystal lattice with adjacent atoms. In such a case, it is more convenient to use the Gibbs energy instead of the chemical potential. The corresponding chemical potentials satisfy the relations

$$
\xi^{0}\left(V_{A}^{0}\right)=H_{V_{A}^{0}}^{0}-T S_{V_{A}^{0}}^{0}=G_{V_{A}^{0}}^{0}
$$

$\xi^{0}\left(A_{A}^{0}\right)=H_{A_{A}^{0}}^{0}-T S_{A_{A}^{0}}^{0}=G_{A_{A}^{0}}^{0}$,

where $S^{0}, H^{0}$ - entropy and enthalpy of structural elements of the crystal lattice under standard conditions.

In the case of a solid, when the specific heats at constant volume and pressure are approximately equal, $c_{P} \approx c_{V}$, the Gibbs energy can be replaced by the free vibration energy $F^{\mathrm{vib}}$ of the corresponding structural elements in the crystal lattice:

$F_{V_{A}^{0}}^{\mathrm{vib}}=U_{V_{A}^{0}}^{\mathrm{vib}}-T S_{V_{A}^{0}}^{\mathrm{vib}} \approx G_{V_{A}^{0}}^{0}$,

$F_{A_{A}^{0}}^{\mathrm{vib}}=U_{A_{A}^{0}}^{\mathrm{vib}}-T S_{A_{A}^{0}}^{\mathrm{vib}} \approx G_{A_{A}^{0}}^{0}$.

Considering that $u_{0}-U_{A_{A}^{0}}^{\mathrm{vib}}=E_{V_{A}^{0}}$ is the defect creation energy, the entropy $S_{A_{A}^{0}}^{\mathrm{vib}}<<S_{V_{A}^{0}}^{\mathrm{vib}}$, and, in the general case, $N\left(A_{A}^{0}\right)=n_{0}$, where $n_{0}=1.48 \cdot 10^{22} \mathrm{~cm}^{-3}$ is the concentration of CdTe lattice sites, we finally present (5) as

$$
N\left(V_{A}^{0}\right)=\frac{n_{0}}{P_{A}} K \exp \left(-\frac{E_{V_{A}^{0}}+U_{V_{A}^{0}}^{\mathrm{vib}}-T S_{V_{A}^{0}}^{\mathrm{vib}}}{k T}\right) .
$$

By executing the simple transformation, we obtain this expression in the standard form as 
$N\left(V_{A}^{0}\right)=\frac{n_{0}}{P_{A}} K \exp \left(\frac{S_{V_{A}^{0}}^{\mathrm{vib}}}{k}\right) \exp \left(-\frac{E_{V_{A}^{0}}+U_{V_{A}^{0}}^{\mathrm{vib}}}{k T}\right)$.

By analogy with (20), it is possible to deduce the formulas for the concentrations of other neutral point defects in chalcogenide:

$$
\begin{aligned}
& N\left(A_{i}^{0}\right)=\frac{P_{A} n_{0}}{K} \exp \left(\frac{S_{A_{i}^{0}}^{\mathrm{vib}}}{k}\right) \exp \left(-\frac{E_{A_{i}^{0}}+U_{A_{i}^{0}}^{\mathrm{vib}}}{k T}\right), \\
& N\left(V_{B}^{0}\right)=\frac{P_{A} n_{0}}{K} \exp \left(\frac{S_{V_{B}^{0}}^{\mathrm{vib}}}{k}\right) \exp \left(-\frac{E_{V_{B}^{0}}+U_{V_{B}^{0}}^{\mathrm{vib}}}{k T}\right), \\
& N\left(B_{i}^{0}\right)=\frac{n_{0}}{P_{A}} K \exp \left(\frac{S_{B_{i}^{0}}^{\mathrm{vib}}}{k}\right) \exp \left(-\frac{E_{B_{i}^{0}}+U_{B_{i}^{0}}^{\mathrm{vib}}}{k T}\right), \\
& N\left(B_{A}^{0}\right)=\frac{n_{0}}{P_{A}^{2}} K^{2} \exp \left(\frac{S_{B_{A}^{0}}^{\mathrm{vib}}}{k}\right) \exp \left(-\frac{E_{B_{A}^{0}}+U_{B_{A}^{0}}^{\mathrm{vib}}}{k T}\right), \\
& N\left(A_{B}^{0}\right)=\frac{n_{0} P_{A}^{2}}{K^{2}} \exp \left(\frac{S_{A_{B}^{0}}^{\mathrm{vib}}}{k}\right) \exp \left(-\frac{E_{A_{B}^{0}}+U_{A_{B}^{0}}^{\mathrm{vib}}}{k T}\right) .
\end{aligned}
$$

The calculation of $E, U^{\mathrm{vib}}$, and $S^{\mathrm{vib}}$ is reduced to modeling the interaction of defects with the crystal lattice by the $a b$ initio method which is based on only the quantum-mechanical and thermodynamic parameters of $\mathrm{Cd}$ and Te atoms. By an example of the formation of point defects in the cadmium sublattice in CdTe and $\mathrm{HgCdTe}$, the appropriate difficult mathematical computations were carried out in [10, 12-15]. Some of them were partially confirmed in a plenty of experiments carried out on CdTe single crystals in $[16,17]$. The results of these researches are presented in Table 1. The energy value designated by the asterisk corresponds to two tetrahedral interstitial sites.

Table 1 shows the essential differences in the values of the thermodynamic functions $E, U^{\mathrm{vib}}$, and $S^{\text {vib }}$ determined by different authors, in particular in the energies of formation of antistructural defects $\mathrm{Te}_{\mathrm{Cd}}^{0}$ and tellurium vacancies $V_{\mathrm{Te}}^{0}$. Therefore, in our opinion, the most reliable results of calculations of the thermodynamic functions of the defect creation process are $E, U^{\text {vib }}$, and $S^{\text {vib }}[16,17]$ which have found a sufficient confirmation by experiment data. We will use them in the modeling in what follows.

If the concentrations of neutral defects are determined, it is easy to find the concentrations of the donors and acceptors of charged point defects by using the Fermi-Dirac statistics and the condition of electroneutrality:
Table 1. Calculated parameters of the creation of neutral point defects using the "ab initio" approach.

\begin{tabular}{|l|c|c|c|}
\hline $\begin{array}{l}\text { Type of } \\
\text { defects }\end{array}$ & $\begin{array}{c}E+U^{\mathrm{vib}}, \\
\Delta H, \mathrm{eV}\end{array}$ & $S^{\text {vib }}, k_{b}$ & References \\
\hline \multirow{4}{*}{$\mathrm{Cd}_{i}^{0}$} & 1.65 & -16.4 & {$[3]$} \\
\cline { 2 - 4 } & $0.96(1.16)^{*}$ & $11.1(16.4)$ & {$[12-13]$} \\
& $1.29(1.36)^{*}$ & & {$[16]$} \\
\cline { 2 - 4 } & $1.97(1.97)$ & $38.46(36.5)$ & {$[18]$} \\
\cline { 2 - 4 } & $2.04(2.26)$ & - & {$[14]$} \\
\hline \multirow{4}{*}{$V_{\mathrm{Cd}}^{0}$} & 3.75 & 5.34 & {$[3]$} \\
\cline { 2 - 4 } & $3.55(3.58)$ & $-5.6(-8.9)$ & {$[16]$} \\
\cline { 2 - 4 } & $3.84(2.11)$ & $-13.05(33.8)$ & {$[18]$} \\
\cline { 2 - 4 } $\mathrm{Te}_{i}^{0}$ & 2.67 & - & {$[14]$} \\
\cline { 2 - 4 } & 1.50 & -19.7 & {$[3]$} \\
\cline { 2 - 4 } & $1.97(1.95)$ & $-38.54(36.5)$ & {$[18]$} \\
\hline \multirow{4}{*}{$V_{\mathrm{Te}}^{0}$} & 3.41 & - & {$[14]$} \\
\cline { 2 - 4 } & $1.41(4.06)$ & $33.83(55)$ & {$[18]$} \\
\cline { 2 - 4 } & 1.57 & 19.7 & {$[3]$} \\
\hline \multirow{3}{*}{$\mathrm{Te}_{\mathrm{Cd}}^{0}$} & 3.24 & - & {$[14]$} \\
\cline { 2 - 4 } & 5.72 & 37.5 & {$[3]$} \\
\cline { 2 - 4 } & $4.72(6.3)$ & $-17(-5.6)$ & {$[16]$} \\
\cline { 2 - 4 } & $-0.81(3.24)$ & $-23.71(68.8)$ & {$[18]$} \\
\cline { 2 - 4 } & 3.7 & - & {$[14]$} \\
\hline \multirow{5}{*}{} & & & \\
\hline
\end{tabular}

$$
n=\frac{N_{c}}{\exp \left[\left(E_{g}-\mu_{\mathrm{F}}\right) / k T\right]+1},
$$

$$
p=\frac{N_{\mathrm{v}}}{\exp \left(\mu_{\mathrm{F}} / k T\right)+1}
$$

$$
N\left(X_{d i}^{z+}\right)=\frac{N\left(X_{d i}^{0}\right)}{g_{d} \exp \left[-\left(E_{g}-E_{d i}-\mu_{\mathrm{F}}\right) / k T\right]+1},
$$

$$
N\left(X_{a i}^{z-}\right)=\frac{N\left(X_{a i}^{0}\right)}{g_{d} \exp \left[\left(E_{a i}-\mu_{\mathrm{F}}\right) / k T\right]+1},
$$

$n+N\left(V_{A}^{-}\right)+N\left(V_{A}^{2-}\right)+N\left(B_{i}^{-}\right)+N\left(B_{i}^{2-}\right)=$

$=p+N\left(A_{i}^{+}\right)+N\left(A_{i}^{2+}\right)+N\left(V_{B}^{+}\right)+N\left(V_{B}^{2+}\right)+N\left(B_{A}^{+}\right)+N\left(B_{A}^{2+}\right)$,

where $N_{c(\mathrm{v})}=2\left(\frac{2 \pi m_{e(p)} k T}{h^{2}}\right)^{3 / 2}-$ effective density of states in the conduction band (valence band); $m_{e(p)}-$ effective mass of an electron $\left(0.35 m_{0}\right)$ or a hole $\left(0.83 m_{0}\right) ; E_{g}-\mathrm{BG}$ energy; $\mu_{\mathrm{F}}-$ Fermi energy; $z-$ degree of defect ionization; $g$ - degeneracy factor; $E_{a}$ and $E_{d}$ - ionization energies of acceptors and donors, respectively; $N\left(X^{0}\right)$ - concentration of neutral defects; $N\left(X_{d}^{z+}\right)$ - concentration of donors; and $N\left(X_{a}^{z-}\right)-$ concentration of acceptors. 
Table 2. Depths of the energy levels of native point defects in the CdTe band gap.

\begin{tabular}{|c|c|c|c|c|c|c|c|c|}
\hline \multirow{2}{*}{$\begin{array}{l}\text { Type of } \\
\text { defects }\end{array}$} & \multicolumn{8}{|c|}{$\Delta E, \mathrm{eV}$} \\
\hline & [8] & [20] & [12-13] & [14] & [16-17] & [18] & [15] & [19] \\
\hline $\mathrm{Cd}_{i}^{+}$ & - & $E_{c}-0.02$ & 0 & $E_{c}-0.33$ & $\begin{array}{c}0^{*} \\
E_{c}-0.21^{*}\end{array}$ & $\begin{array}{c}0 \\
\text { from [12] }\end{array}$ & $E_{c}-0.80$ & - \\
\hline $\mathrm{Cd}_{i}^{2+}$ & $E_{c}-0.15$ & $E_{c}-0.56$ & $E_{c}-0.20$ & $\begin{array}{l}E_{c}-0.46^{*} \\
E_{c}-0.56^{*}\end{array}$ & $\begin{array}{l}E_{c}-0.17 * \\
E_{c}-0.36^{*}\end{array}$ & $\begin{array}{c}E_{c}-0.20 \\
\text { from [12] }\end{array}$ & - & $\begin{array}{l}E_{c}-0.54 \\
E_{c}-0.56 \\
E_{c}-0.61 \\
E_{c}-0.64 \\
E_{v}+0.88\end{array}$ \\
\hline$V_{\mathrm{Te}}^{+}$ & - & - & $E_{c}-0.40$ & - & - & $\begin{array}{c}E_{c}-0.40 \\
\text { from [12] }\end{array}$ & $\begin{array}{c}E_{c}-0.01 \\
E_{c}-1.1 \\
E_{c}-1.2\end{array}$ & - \\
\hline$V_{\mathrm{Te}}^{2+}$ & $E_{c}-0.035$ & $E_{c}-0.58$ & $E_{c}-0.50$ & $E_{c}-0.71$ & - & $\begin{array}{c}E_{c}-0.50 \\
\text { from [12] }\end{array}$ & $\begin{array}{c}E_{c}-0.05 \\
0\end{array}$ & - \\
\hline $\mathrm{Te}_{\mathrm{Cd}}^{+}$ & - & - & 0 & $E_{c}-0.34$ & $E_{c}-1.44$ & $\begin{array}{c}0 \\
\text { from [12] }\end{array}$ & $\begin{array}{c}E_{c}-0.60 \\
0\end{array}$ & - \\
\hline $\mathrm{Te}_{\mathrm{Cd}}^{2+}$ & - & - & $E_{c}-0.40$ & $E_{c}-0.59$ & $E_{c}-1.6$ & $\begin{array}{c}E_{c}-0.40 \\
\text { from [12] }\end{array}$ & $E_{c}-1.0$ & - \\
\hline$V_{\mathrm{Cd}}^{-}$ & $E_{v}+0.05$ & $E_{v}+0.05$ & $E_{v}+0.20$ & $E_{v}+0.13$ & $\begin{array}{l}E_{v}+0.05 \\
E_{v}+0.20\end{array}$ & $\begin{array}{l}E_{v}+0.20 \\
\text { from [12] }\end{array}$ & $E_{v}+0.05$ & - \\
\hline$V_{\mathrm{Cd}}^{2-}$ & $E_{v}+0.90$ & $E_{v}+0.66$ & $E_{v}+0.80$ & $E_{v}+0.21$ & $E_{v}+0.47$ & $\begin{array}{l}E_{v}+0.80 \\
\text { from [12] }\end{array}$ & $\begin{array}{l}E_{v}+0.47 \\
E_{v}+0.60 \\
E_{v}+0.76\end{array}$ & $\begin{array}{l}E_{v}+0.18 \\
E_{v}+0.33 \\
E_{v}+0.38 \\
E_{v}+0.49 \\
E_{v}+0.51 \\
E_{v}+0.54 \\
E_{v}+0.60 \\
E_{v}+0.62 \\
E_{v}+0.63 \\
E_{v}+0.65\end{array}$ \\
\hline $\mathrm{Te}_{i}^{-}$ & $E_{v}+0.15$ & $E_{v}+0.15$ & - & $E_{v}+0.67$ & - & $E_{v}+0.10$ & $E_{v}-0.70$ & - \\
\hline $\mathrm{Te}_{i}^{2-}$ & - & - & - & $E_{v}+0.74$ & - & $\begin{array}{l}E_{v}+0.60^{*} \\
E_{v}+0.70^{*}\end{array}$ & $E_{v}-1.0$ & - \\
\hline
\end{tabular}

The ionization energy of acceptors and the location of the Fermi level are reckoned from the top of the valence band, and the ionization energy of donors is counted off the bottom of the conduction band. As the factors of spin degeneracy of levels, we take $g=2$ for single-charged donors of defects and $g=1 / 2$ for acceptor levels, doubly charged centers, according to [17].

At present, the spectrum of energy levels of point defects in the BG of cadmium telluride is not enough studied, and the identification of the majority of them is disputable. The newest works on the determination of the depth of local centers $\Delta E$ of a different nature were systematized in [18]. Only the method of photoinduction of currents gave more than one hundred fifty deep energy levels. Most of them are caused just by native point defects. Some hope to unequivocally determine the energy $\Delta E$ of native defects is given by theoretical works [12-14], where the depth of located centers was found from the "first principles". Unfortunately, these methods are rather inexact. The results of some works, where the energy levels of point defects in chalcogenide are theoretically or experimentally determined, are systematized in Table 2.

The calculation of the concentration of neutral defects in CdTe single crystals and thin films was carried by us with the help of Eqs. (21)-(26) with regard for the values of the thermodynamic functions of the 


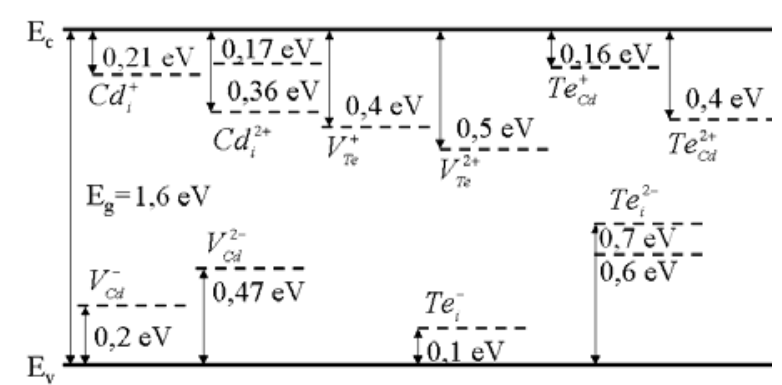

Fig. 1. Location of defect energy levels in the CdTe band gap.

creation of defects given in Table 1 . We have used the model which takes into account the most complete collection of defects in chalcogenide. It includes defects in the cadmium $\left(V_{\mathrm{Cd}}^{0}-\right.$ cadmium vacancy, $\mathrm{Cd}_{i}^{0}-$ cadmium interstitial atom) and tellurium sublattices ( $V_{\mathrm{Cd}}^{0}-$ tellurium vacancy, $\mathrm{Te}_{i}^{0}-$ tellurium interstitial atom), as well as the existence of antistructural defects $\mathrm{Te}_{\mathrm{Cd}}^{0}$. The antistructural defect $\mathrm{Cd}_{\mathrm{Te}}^{0}$ was not taken into account, as its existence in a material is improbable [12,
13]. Then we calculated the Fermi level location and the concentration of ionized defects according to Eqs. (27)(31). The type of defects and the positions of energy levels which were used in the modeling are presented in Fig. 1.

We compared the results of modeling with the data obtained with the use of the traditional approach for a number of models, which have found a wide application now.

The results of modeling of PDE in single crystals during annealing at different cadmium pressures are given in Fig. 2, and Fig. 3 presents the result of calculations of the Hall charge carrier concentration in samples.

Figure 3 shows that the different models and sets of QE constants offered in $[2,6,9]$ at high cadmium vapor pressures give a rather good correlation between the results of modeling and the experimental value of $n$. It is well clear, because the corresponding QE constants were obtained as a result of optimization of the solution of the multifactor task just in the range of high $P_{\mathrm{Cd}}$. At a lower cadmium pressure (in a material enriched by tellurium), the large distinction is observed between the results of modeling within different models, which requires making a choice of the most reliable ones.
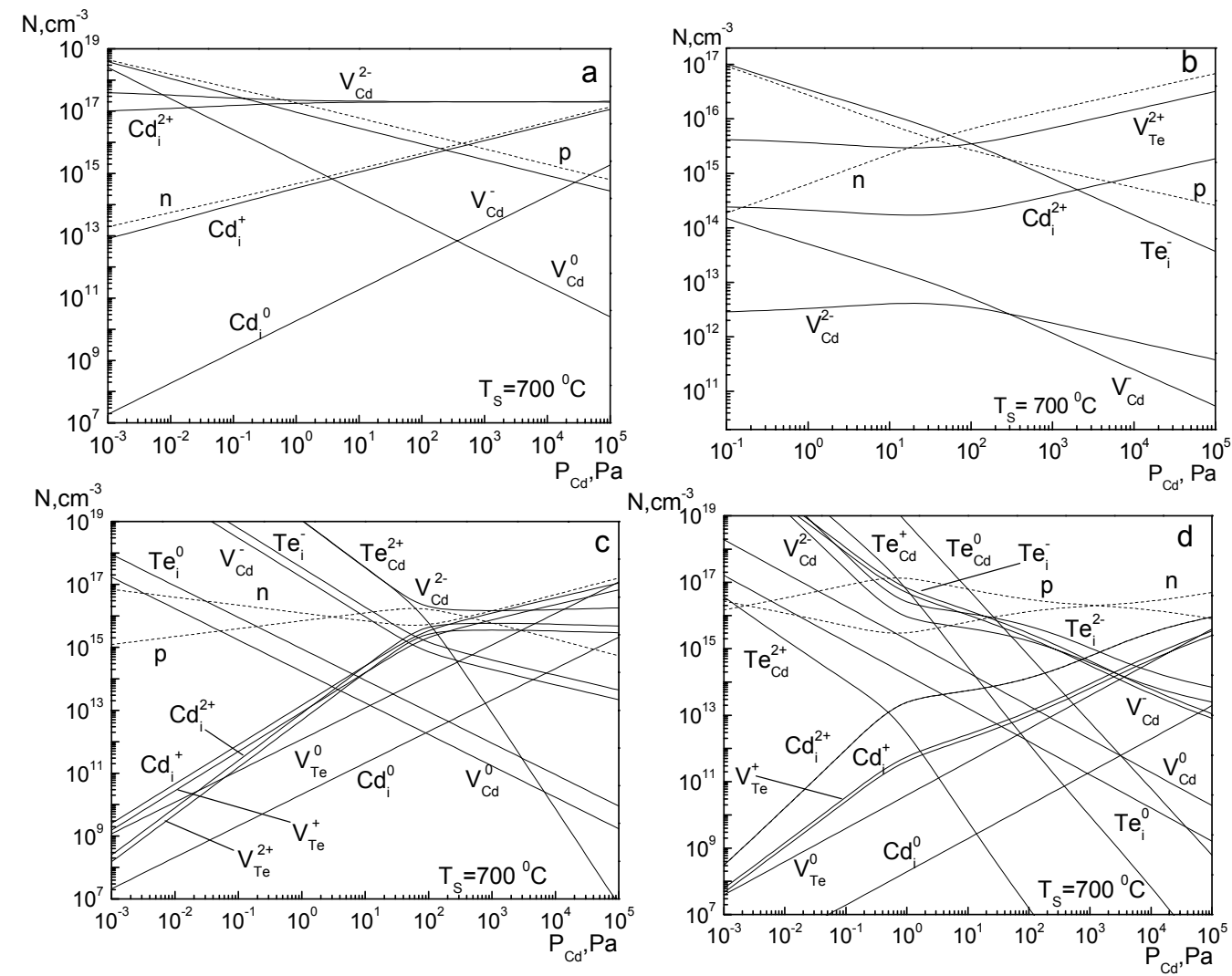

Fig. 2. Concentration of native point defects and free charge carriers under different partial pressures of cadmium vapor $P_{C d}$ at the annealing temperature of CdTe single crystals. Calculations within the traditional approach: $a$ and $b$ correspond to models in [2] and [6], respectively; $c$ - complete sets of defects [9]. Calculations within the "first principles" approach: $d$. 


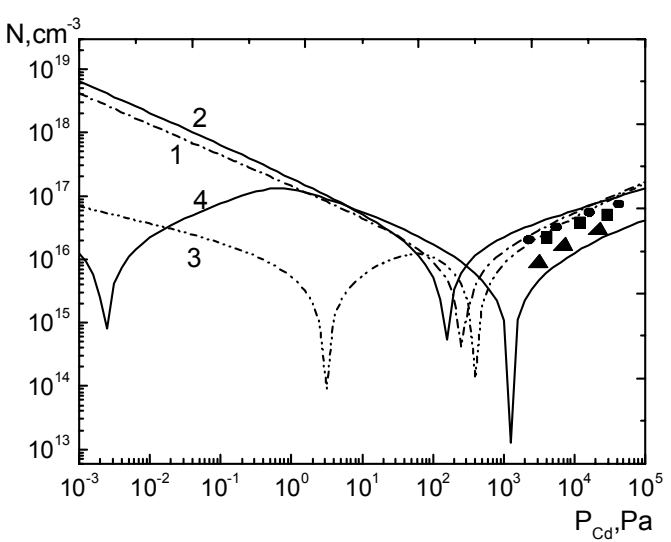

Fig. 3. Hall concentration of free charge carriers at different partial pressures of cadmium vapor in CdTe single crystals and the annealing temperature $T_{s}=700{ }^{\circ} \mathrm{C}$. Calculations within the traditional approach: 1 and 2 correspond to models in [2] and [6], respectively; $c$ - complete sets of defects [9]. Calculations within the "first principles" approach: $d$. Experimental data are taken from $[8,16,21]$.
In Fig. 4, we present the results of modeling of PDE in a material from the "first principles" during the annealing of CdTe single crystals and in thin films on their fabrication under different technological conditions in the cases of full equilibrium and quenching [2,9]. In addition, we display the results of calculations of a position of the Fermi level in a material.

It is obvious from Figs. 2 and 4 that the dominant defects in single crystals at high cadmium pressure and annealing are doubly charged tellurium vacancies (Fig. 2, b) or interstitial cadmium atoms (Fig. 2, c, d) in agreement with the experimental data $[8,16,17,20]$. Just such defects can ensure the dependence of the concentration of free carriers on the cadmium pressure as $n \sim P_{\mathrm{Cd}}^{1 / 3}$. A type of predominant defects in the material enriched with tellurium is determined by the chosen model. As a rule, they are $\mathrm{Te}_{i}^{-}$or $\mathrm{Te}_{\mathrm{Cd}}^{+}\left(\mathrm{Te}_{\mathrm{Cd}}^{2+}\right)$.
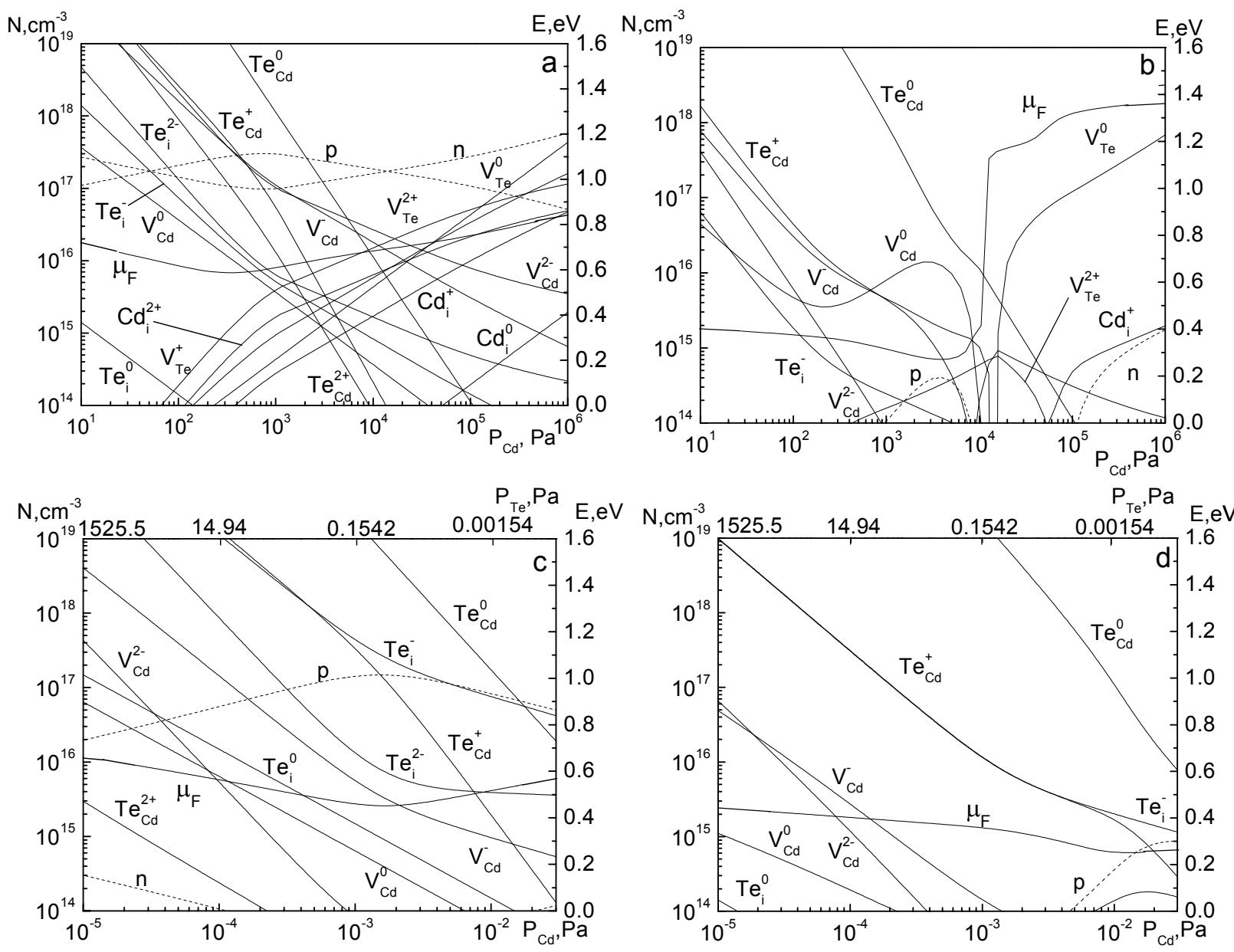

Fig. 4. Concentration of native point defects and the Fermi level location at different partial pressures of cadmium vapor: $a, b-$ CdTe single crystal annealing $\left(T_{s}=900^{\circ} \mathrm{C}\right)$, full equilibrium and quenching respectively; $c, d$-condensation of CdTe thin films full equilibrium $\left(T_{s}=550{ }^{\circ} \mathrm{C}, T_{e}=600-1000^{\circ} \mathrm{C}\right)$ and quenching $\left(T_{s}=20^{\circ} \mathrm{C}, T_{e}=600-1000^{\circ} \mathrm{C}\right)$, respectively. 


\section{Conclusions}

The results of calculations presented in this work allow choosing the technological conditions for the fabrication of single crystals and CdTe thin films with the predetermined structure of point defects and electrophysical properties.

The offered model is the most complete among possible ones and, with regard for the corresponding constants of materials, can be used for the modeling of PDE in any $\mathrm{A}_{2} \mathrm{~B}_{6}$ compound. Thus, the problem of the choice of models adequate to the experimental data is reduced to the determination of the defect creation energy of uncharged defects and the position of the energy levels of charged defects.

\section{References}

1. D.V. Korbutyak, S.V. Melnichuk, Cadmium Telluride Impurity Defects States and Detector Properties. Ed. Ivan Federov, p. 7-30, Kyiv, 2000 (in Ukrainian).

2. F.A. Kreger, The Chemistry of Imperfect Crystals. North-Holland, Amsterdam, 1964, p. 467-489.

3. Q. Chen, M. Hillert, Phase equilibria, defect chemistry and semiconducting properties of CdTe - thermodynamic models // J. Solid State Chem. 27(8), p. 961-971 (1998).

4. V.M. Glazov, L.M. Pavlova, Homogeneity region in cadmium telluride system // Neorg. Mater. 30(5), p. 629-634 (1994) (in Russian).

5. D.M. Freik, V.V. Prokopiv, U.M. Pusklunets, Atomic defects and their compensation in doped and undoped cadmium telluride //Fiz. Khim. Tverdogo Tela 3(4), p. 547-554 (2002) (in Russian).

6. P.M. Fochuk, O.E. Panchuk, L.P. Sherbak, Dominant point defects in CdTe single crystal: Cd saturation region // Ibid. 5(1), p. 136-141 (2004).

7. D. Nobel, Phase equilibria and conducting properties of cadmium telluride // J. Philips Res. Repts 3(14), p. 430-492 (1959).

8. S. Chern, F. Kreger, The defects structure of CdTe // J. Solid State Chem. 14, p. 33-43 (1975).
9. V.V. Kosyak, A.S. Opanasyuk, I.Yu. Protsenko. Point defects ensemble in CdTe single crystals and films in the case of full equilibrium and quenching // Funkts. Mat. 12(4), p. 797-806 (2005).

10. M.A. Berding, M. Schilfgaarde, A. Sher, Firstprinciples calculation of native defect densities in $\mathrm{Hg}_{0.8} \mathrm{Cd}_{0.2}$ Te // Phys. Rev. B 50(3), p. 1519-1534 (1994).

11. R.F. Brebrick, Equilibrium constants for quasichemical defect reactions // J. Electr. Mater. 33(11), p. 24-26 (2004).

12. M.A. Berding, M. Schilfgaarde, A.T. Paxton, A. Sher, Defects in ZnTe, CdTe and HgTe: Total energy calculations // J. Vac. Sci. Technol. A 8(2), p. 1103-1107 (1990).

13. M.A. Berding, Native point defects in CdTe // Phys. Rev. B 60 (12), p. $8943-8950$ (1999).

14. S.H. Wei, S.B. Zhang, Theoretical trends of defect formation and doping limits in II-VI semiconductors: The case of CdTe // Phys. Rev. B 60, 155211 (2002).

15. V. Babentsov, V. Corregidor, K. Benz, M. Fiederle, T. Feltgen, E. Dieguez, Defect engineering in CdTe, based on the total energies of elementary defects // Nucl. Instr. Meth. Phys. Res. A 458, p. 85-89 (2001).

16. J. Franc, P. Hoschl, R. Grill, L. Turjanska, E. Belas, P. Moravec, Galvanomagnetic properties of CdTe below and above the melting point // J. Electr. Mater. 30(6), p. 595-602 (2001).

17. R. Grill, A. Zappettini, Point defects and diffusion in cadmium telluride // Progr. Crystal Growth and Characterization of Materials 48(49), p. 209-244 (2004).

18. L. Yujie, M. Guoli, J. Wanqi, Point defects in CdTe // J. Cryst. Growth 256, p. 266-275 (2003).

19. X. Mathew, Photo-induced current transient spectroscopic study of the traps in CdTe // Solar Energy Materials \& Solar Cells 76, p. 225-242 (2003).

20. S.N. Maksimovskiy, S.N. Kobeleva, Predominant point defects in CdTe // Neorg. Mater. 22(6), p. 923-925 (1986) (in Russian).

21. F.T.J. Smith, Electrically active point defects in cadmium telluride // J. Metallurg. Trans. 1(3), p. 617-621 (1970). 